\title{
Communication at risk
}

\section{The L'Aquila earthquake trial tragically highlights that risk communication is integral to Earth science training.}

In October 2012, six Italian scientists and one government official were found guilty of the manslaughter of 29 people who died during the 2009 L'Aquila earthquake. Each was sentenced to six years in prison. When the case was first opened, geological societies rushed to the aid of the scientists - a natural reaction to their colleagues being under attack. However, as disaster risk scientist David Alexander argued in a seminar at University College London in January 2013, on closer inspection of the trial and the evidence presented, there could be a case for negligence.

Importantly, the levy against the accused was that of failing to adequately evaluate and communicate risk and presenting falsely reassuring findings to the public, not of failing to predict the quake, as was often assumed after the trial was announced. Comments made by Bernardo De Bernardinis, the government official on trial and deputy head of Italy's Civil Protection Department at the time, could be interpreted as falsely reassuring. Before the earthquake, De Bernardinis stated publicly that ongoing seismic tremors affecting L'Aquila posed no danger, and that the scientific community continued to confirm to him that the situation was favourable. During the trial, several witnesses testified to say that comments from officials lulled the community into a false sense of security. According to the prosecution, some people remained in their homes while the ground shook, whereas ordinarily they would have evacuated, and were killed when the buildings collapsed (Science 338, 184-188; 2012).

The case for inadequate evaluation and communication of risk by the scientists is less obvious. The scientists had explained to the officials that numerous seismic swarms in the past hadn't culminated in large earthquakes and the probability of one on this occasion was low (Science 338, 184-188; 2012). This assessment and communication to the officials seems fair. Why then did the scientists not publicly step forward to correct the overly reassuring statements made by De Bernardinis and to reiterate that there was still an ongoing possibility, even if slim, of an earthquake? Earth scientists should know that the communication of hazard assessments requires utmost care and attention to uncertainty, yet it seems that these scientists didn't speak directly to the public at any point. Perhaps they should have done.

Whether there is a case for negligence or not, the charge of manslaughter is extreme. Such a severe sentence will make scientists hesitant to advise and is unlikely to improve communication. To avoid similar shortfalls in the future, we should focus on equipping Earth scientists with adequate communication skills, and heighten their understanding of how their words - or those of government officials speaking to the public on their behalf - will be perceived. Risk communication and media training are common in many fields: doctors, athletes and even models are groomed on how to deliver an appropriate response during press interrogation.

Earth scientists inform on life-ordeath situations, from impending volcanic eruptions and seismic hazards, to flood or tornado warnings and climate change. It is vitally important that those with hazardrelated expertise learn and practice early on how to phrase recommendations so that the public gets the right message.

\section{Expanding spheres of interest}

\section{Extrasolar planet research is booming. We welcome submissions with links to the geosciences.}

Much can be learned about the planets of the Solar System using geoscience knowledge, methods and reasoning. Similarly, investigations of known geophysical phenomena in a different setting - for example, rainfall in the hydrocarbon atmosphere of Saturn's moon Titan - can bring fresh insights for Earth.

To foster such a cross-fertilization of ideas, we at Nature Geoscience have welcomed papers from the planetary sciences since the journal launch. With the ongoing and rapid expansion of information on extrasolar planets, in terms of both the number of known exoplanets and their characterization, we are pleased to extend the invitation to scientists studying planets outside the Solar System, as long as the reasoning is rooted in the geosciences.
A true exploratory buzz of excitement is accompanying the discovery of more - and stranger - planets outside the Solar System. The flurry of search activities started a few years ago in the field of astronomy, which provides the tools for the identification of exoplanets. At the January 2013 meeting of the American Astronomical Society in Long Beach, California, extrasolar planets featured on most days of the programme. But Earth and planetary scientists have been quick to take the bait. For example, a Chapman conference (American Geophysical Union) in June 2013 will be dedicated to discussing the atmosphere of the Earth, the Solar System planets and exoplanets (http:// chapman.agu.org/planetaryatmospheres/).

Most exciting, from an Earth and planetary science perspective, is that astronomers are not only discovering planets that circle other stars, but are beginning to also glean sufficient information about their size, mass and orbit to allow geophysical analyses into composition, climate and circulation. A Commentary on page 81 portrays a number of candidate planets and planet-star configurations that could lend themselves to geoscientific study.

The most eagerly pursued question in exoplanet research is that of habitability. Humans will always want to know if there is life out there in the Universe (and if so, where and in what circumstances). Answering questions may take a while. In the mean time, the sheer number and variety of exoplanets will allow us to test our ideas about the laws of geophysics in unimagined ways. At Nature Geoscience, we look forward to reading about that journey of discovery. 